\title{
On the Effectiveness of Digital Contact Tracing and Contact Prevention Under Varying COVID-19 Infection Detection Rates
}

\section{German José Soldano}

Departamento de Química Teórica y Computacional, Facultad de Ciencias Químicas, Universidad Nacional de Córdoba / INFIQC-CONICET

\section{Juan Andrés Fraire}

Facultad de Ciencias Exactas, Físicas y Naturales, Universidad Nacional de Córdoba / IDIT-CONICET Jorge Manuel Finochietto

Facultad de Ciencias Exactas, Físicas y Naturales, Universidad Nacional de Córdoba / IDIT-CONICET

Rodrigo Quiroga ( $\nabla$ rquiroga@unc.edu.ar)

Departamento de Química Teórica y Computacional, Facultad de Ciencias Químicas, Universidad Nacional de Córdoba / INFIQC-CONICET

\section{Research Article}

Keywords: SARS-CoV-2, contact tracing, SERIA, Covid-19

Posted Date: February 10th, 2021

DOl: https://doi.org/10.21203/rs.3.rs-179393/v1

License: (c) (1) This work is licensed under a Creative Commons Attribution 4.0 International License. Read Full License

Version of Record: A version of this preprint was published at Scientific Reports on July 13th, 2021. See the published version at https://doi.org/10.1038/s41598-021-93538-5. 


\title{
On the Effectiveness of Digital Contact Tracing and Contact Prevention Under Varying COVID-19 Infection Detection Rates
}

\author{
Germán J. Soldano ${ }^{1,3}$, Juan A. Fraire ${ }^{2,4,5}$, Jorge M. Finochietto ${ }^{2,4}$, and Rodrigo \\ Quiroga $a^{1,3, *}$
}

\author{
${ }^{1}$ Instituto de Investigaciones en Fisico-Química de Córdoba (INFIQC-CONICET) \\ ${ }^{2}$ Instituto De Estudios Avanzados En Ingenieria Y Tecnología (IDIT-CONICET) \\ ${ }^{3}$ Universidad Nacional de Córdoba, Facultad de Ciencias Químicas, Argentina \\ ${ }^{4}$ Universidad Nacional de Córdoba, Facultad de Ciencias Exactas, Físicas y Naturales, Argentina \\ ${ }^{5}$ Saarland University, Saarland Informatics Campus, Saarbrücken, Germany \\ *Corresponding author, email: rquiroga@unc.edu.ar
}

\begin{abstract}
A plethora of measures are being combined in the attempt to reduce SARS-CoV-2 spread. Due to its sustainability over time, contact tracing is one of the most frequently applied interventions worldwide, albeit with mixed results. In this work, we evaluate the perfomance of contact tracing for different infection detection rates and response time delays. We also introduce and analyze a novel strategy we call contact prevention. We model the effect of both strategies on contagion dynamics in SERIA, an agent-based simulation platform that implements realistic population-dependent statistical distributions. Results show that diagnostic/response time delays and low infection detection rates greatly impair the effect of contact tracing strategies, while contact prevention remains effective in these scenarios. Therefore, contact prevention could play a significant role in pandemic mitigation, specially in under-developed countries where diagnostic and tracing capabilities are insufficient. Contact prevention could thus sustainably reduce the propagation of respiratory viruses while relying on available technology, respecting data privacy, and most importantly, promoting community-based awareness and social responsibility. Depending on infection detection and app adoption rates, applying a combination of contact tracing and contact prevention could reduce pandemic-related mortality by $20 \%-56 \%$.
\end{abstract}

\section{Introduction}

The COVID-19 pandemic has challenged health authorities around the world since December 2019. Many governments immediately implemented physical distancing and self-isolation measures, ranging from simple "stay-at-home" recommendations to strict lock-downs ${ }^{1}$. Although straightforward, fast and effective in controlling the propagation of the virus, rigorous lock-downs are emergency measures which imply profound economical and social consequences, and cannot be sustained over long periods of time, specially in underdeveloped and developing countries. Sustainable and widely applied nonpharmaceutical interventions such as effectively communicating prevention measures, cancellation of large-scale public gatherings, widespread mask utilization, and travel restrictions, proved to be insufficient to contain viral spread in many countries ${ }^{2}$.

In this context, Contact Tracing (CT) has been extensively used to attempt to control outbreaks ${ }^{3}$ by identifying and isolating close contacts of diagnosed patients as soon as possible, to prevent further contagion. However, the efficiency of the approach in diminishing COVID-19 propagation strictly depends on how quickly, broadly, and accurately the contact tracing process is ${ }^{4}$. In particular, it is crucial to minimize delays in diagnostics, contact determination and detection, as well as the subsequent isolation of all possibly infected individuals ${ }^{5}$. This argues in favor of so-called digital CT, where smartphones automatically store and report contact information ${ }^{3}$ using mainly Bluetooth Low Energy (BLE) technology for proximity detection between devices $^{6}$. The effectiveness of CT has been enhanced by embracing this technology in several countries ${ }^{7,8}$, although not free of data privacy concerns, among other controversies 9,10 .

Both manual and digital CT evidenced a common disadvantage intrinsic to the very nature of this reactive strategy: it depends largely on the percentage of infected individuals which are successfully and quickly diagnosed with COVID-19. However, this issue has been scarcely documented and noted by the community, even though infection detection rates are estimated to be below $12 \%$ for most countries, and $16 \%$ or less even for developed countries such as the United States of America, Canada, China, Sweden and The United Kingdom ${ }^{11}$. Accordingly, an analysis for the city of New York estimates an IDR of $15-20 \%{ }^{12}$. Undetected infections are a key characteristic of the COVID-19 pandemic that severely impacts CT 
strategies, as no contact tracing is possible without diagnosis, which is most generally triggered by symptom onset. We argue this CT limitation is the main reason for observing satisfactory results only when combined with other policies such as detection and isolation via enhanced/random testing or contact avoidance via household quarantine ${ }^{13}$.

In this work, we analyze the impact of different IDRs and time delays on the effectiveness of CT. With the aim of reducing the dependency on these factors, while improving data privacy, we introduce community-based Contact Prevention (CP). CP is a novel strategy that attempts to diminish viral transmission by warning users from infection risks due to their current social activity. To quantitative asses CT and CP in realistic COVID-19 scenarios, a detailed COVID-19 simulation model based on agents, which we named SERIA, is presented. This model leverages several COVID-19 statistical distributions such as social and household contact profiles, infection detection rates (IDR), population age, viral latency period, and fatality rates. We then evaluate and compare the effect of CP and CT strategies on final epidemic size (FES) and mortality.

\section{Methods}

SERIA is a Monte Carlo agent-based model that reproduces the essential aspects of social and household contacts in the context of COVID-19 ${ }^{1}$. The model includes all agents of the well-known SEIR models ${ }^{14}$, and adds an asymptomatic agent, a fundamental component of the COVID-19 pandemic ${ }^{15}$. Moreover, SERIA is able to evaluate the performance of CT and CP in terms of FES and mortality rate. Simulations of $1 \times 10^{5}$ agents modeling social and household interaction are performed. Social and family contagion are handled separately. Essential aspects of the model are summarized below; detailed explanations, algorithms and validation analysis are given in the Supplementary Information.

Agents We use age as the main defining feature for agents, since it affects all other agent features. For instance, lethality amongst infected, as given by the Infection Fatality Rate (IFR) (see figure S4), and social contact patterns S7. Age is assigned randomly to each agent following a probability distribution given by $P_{\text {age }}$, shown in figure 1 . This function was fitted from the latest Argentinian census conducted in 2012.

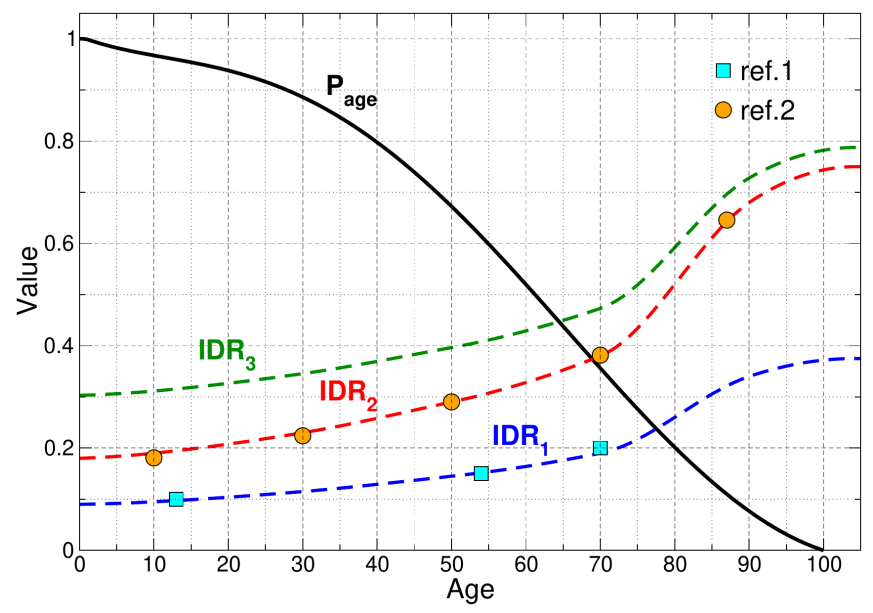

Figure 1. Age distribution function $P_{\text {age }}$ and IDR as a function of age for the three scenarios considered in this work: one in which half the symptomatic are detected (IDR 1$)$, one in which all of them are $\left(\mathrm{IDR}_{2}\right)$, and one in which all the symptomatic and $15 \%$ of the asymptomatic (for each age) are detected (IDR 3 ). Ref.1: ${ }^{12}$ and ref.2: ${ }^{16}$.

Families Household sizes from 1 to 8 are built associating agents so that a given distribution of family sizes and age classes per family is fulfilled. In this case the distribution corresponds to the Argentinian census conducted in 2012. The proportion of homes with 1 to $8+$ members are: $18 \%, 23 \%, 20 \%, 18 \%, 10 \%, 6 \%, 2 \%$ and 3\%, correspondingly. Children, adults and elderly distributions in families are given in figure S6.

Periods Once infected, agents sequentially transit the latency and infection periods, being able to spread the virus only in the latter. The length of the latency period is assigned randomly to each agent, obeying a log-normal distribution that varies between 2 and 14 days $^{17}$, peaking at around 5.5 days $^{18}$. The infection period was set to 12 days with a peak at 1.5 days ${ }^{19}$. Functions controlling these periods are given in figure S5.

Family interaction Household interactions are modeled by simulating contacts between all family members once a day.

\footnotetext{
${ }^{1}$ SERIA is open-source and publicly available in https://bitbucket.org/juanfraire/seria
} 
Social interaction Three fundamental aspects of social contact patterns ${ }^{20}$ are contemplated in the model: i) Young adults have the largest contact frequency, ii) chances of contact decay with the age difference, iii) this decay speed decreases with age and reaches higher values as age increases. We achieve this by sorting contacts candidates and accepting them with a probability $P_{s o c}$ that responds to the above social patterns. The number of accepted contacts is also used as a metric of time.

Contagion If an infected (symptomatic or asymptomatic) and a susceptible agent get in contact, contagion occurs with probability $P_{c t g}$. The latter changes during the infection period following a log-normal distribution ${ }^{21}$, peaking at 1.5 days. $P_{c t g}$ is multiplied by 0.1 in case the infected agent is isolated.

IDR scenarios Although still debated, studies agree that IDR increases with age, and some studies estimate IDR can be as low as $10 \%$ for youngsters and $40 \%$ for the elderly even in wealthy cities, within wealthy countries as is the case of New York ${ }^{12}$. In this context, three IDR scenarios were considered, all with a symptomatic proportion of total infections corresponding to Poletti's results ${ }^{16}$. Symptomatic agents are assumed to self-isolate on symptoms onset.

1. IDR ${ }_{1}$ : (13\% on average) Corresponds to the median IDR estimated for 91 countries $^{11}$. We model this scenario by detecting only half of the symptomatic cases.

2. $\mathrm{IDR}_{2}$ : ( $26 \%$ on average) This IDR is only reported for 18 out of the 91 countries $^{11}$. In SERIA, corresponds to detecting all symptomatic agents.

3. IDR IDR $_{3}$ (37\% on average) Only Australia, Russia, Puerto Rico, Thailand, South Korea and Israel are estimated to have IDRs above $37 \%^{11}$. We model this scenario by detecting all symptomatic and $15 \%$ of asymptomatic infections.

Contact Tracing SERIA comprises manual contact tracing (mCT), which is applied to all reported cases, and digital contact tracing $(\mathrm{dCT})$, which only applies to those with an installed smartphone app. While mCT strictly depends on the IDR, dCT also depends on app adoption rates $(A)$; furthermore, the contact detection effectiveness differs: we use $40 \%$ for mCT, and $85 \%$ for $\mathrm{dCT}^{22,23}$. CT comprises both $\mathrm{mCT}$ and $\mathrm{dCT}$, and it is affected by the time delay $D$, which corresponds to the days from symptom onset of a positive case to the isolation of contacts. In SERIA, all detected contacts of the primary case are automatically isolated $D$ days after symptoms onset. Finally, we also evaluate recursive $\mathrm{CT}(\mathrm{rCT})$, where indirect contacts are also traced ${ }^{24,25}$.

Contact Prevention $\mathrm{CP}$ is possible only using digital means; thus, like dCT, it depends on $A$ and we consider $85 \%$ contact detection efficiency. In contrast with CT, where contacts must be able to be linked to an identity, CP contacts only need to be counted. The average number of daily contacts of each user is then compared with the contact threshold $C_{\max }$ recommended by the authorities through the app. If it is higher, a warning message is sent. App users ideally respond to the warning by lowering their contact frequency to a fraction of their regular contact rate. Such fraction is assigned randomly to agents using a Gaussian distribution. Once the average contact count gets below $C_{\max }$, the warning expires and the user returns to its normal social habits.

We study the performance of CP for $C_{\max }$ from $40 \%$ to $90 \%$ the maximum average of daily close contacts among age groups (1.5 to 3.5 contacts per day, correspondingly). Each household contact is fractionally counted to avoid members of large families reaching the threshold with few social contacts. In the rCP strategy, the app can also count indirect contacts and warn their users if they reach a recursive contact threshold $r C_{\max }$.

\section{Results}

To assess the effectiveness of each strategy in diminishing viral propagation, we perform 365 days of SERIA simulation with initial $\mathrm{R}_{e}=1.5$, and assess the percentage of the population infected at the end of said simulations (FES). $\mathrm{R}_{e}=1.5$ is an estimated $\mathrm{R}_{e}$ for populations that are implementing mandatory mask use, have closed places of worship, schools and universities, banned social gatherings of more than 10 people and implemented protocols for restaurants, bars and gymnasiums. Scenarios analyzed are organized following the aforementioned IDRs distributions, namely 13\%, 26\% and 37\% (overall percentages). For each scenario, the performance of CT and CP strategies are compared for different delays $(D)$ and contact thresholds $\left(C_{\max }\right)$, respectively. The effects of app adoption rates $(A)$ are also studied together with the implementation of a combined $\mathrm{CT}+\mathrm{CP}$ strategy.

\section{Effect of IDR and delay on CT and rCT}

Figure 2 presents the impact of app adoption rates and delays on the effectiveness of CT for each of the IDR scenarios.

The most significant result is the strong effect of IDR on CT effectiveness. For the IDR $_{1}$ scenario, CT hardly reduces viral propagation, even for high app adoption rates and low delays. Therefore, adequate diagnostic testing is a requirement for effective CT. CT effectiveness increases for IDR 2 , and most notably, for IDR 3 . These results could explain the limited success 
(a)

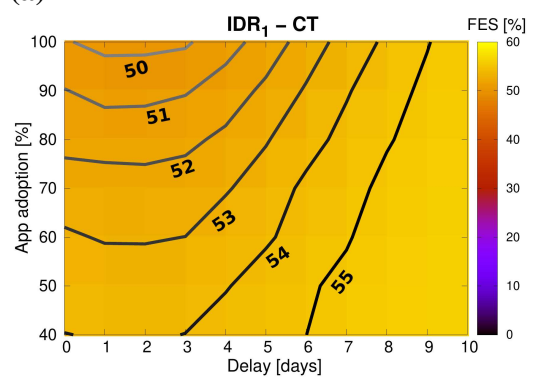

(d)

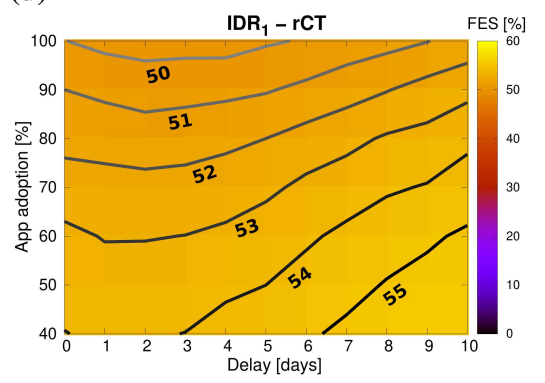

(b)

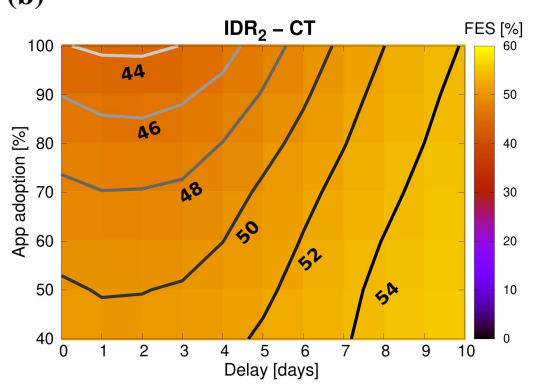

(e)

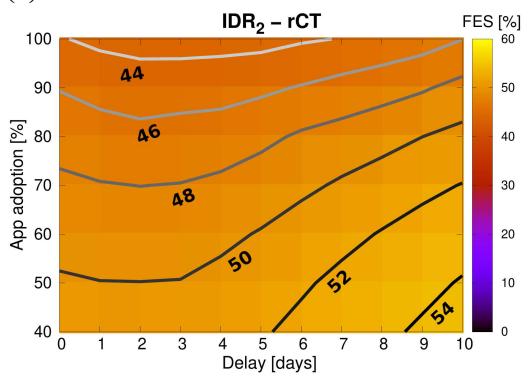

(c)

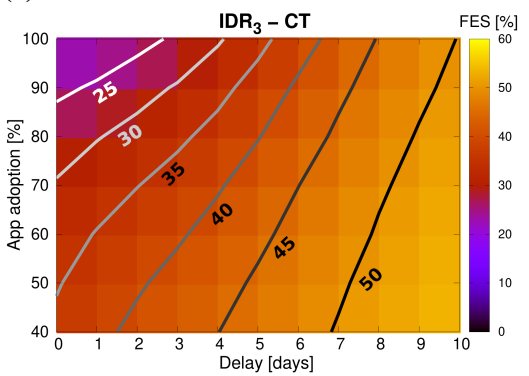

(f)

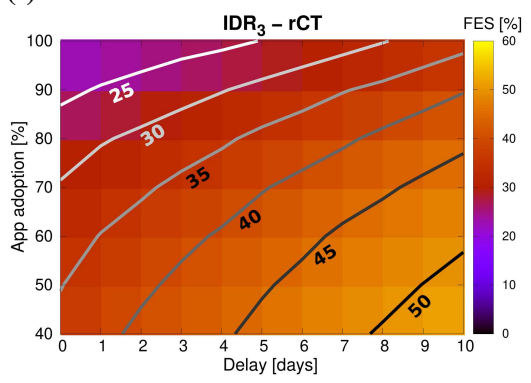

Figure 2. Final epidemic size shown as a heatmap, as a function of CT app adoption and delay (days from symptom onset to contact isolation). The performance of CT (above) and rCT (below) are compared for the three IDR scenarios detailed in figure 1. Contour lines for different FES values are shown. Greater delays, low app adoption, and low IDRs result in high FES values, which indicate low CT effectiveness.

observed for digital CT techniques in reducing the spread of COVID-19 in the first half of $2019^{26}$, when diagnostic capabilities were still low (with IDRs similar to, or even lower than, IDR $_{1}$ ). The impact of time delays on rCT effectiveness is lower than for CT. At low delays, however, both CT and rCT show practically the same performance for different app adoptions. Thus, rCT may result particularly convenient in cases with high diagnostic/isolation delays.

\section{CT versus $\mathrm{CP}$ and combined strategies}

Figure 3 shows the performance of $\mathrm{CT}, \mathrm{CP}$ and $\mathrm{CT}+\mathrm{CP}$ strategies for $\mathrm{IDR}_{1}, \mathrm{IDR}_{2}$ and $\mathrm{IDR}_{3}$ scenarios. To this end, we have fixed parameters to a delay of 3 days in CT and a contact threshold of $C_{\max }=3.1 \mathrm{in} \mathrm{CP}$. The rather low value of $D$ is quite optimistic, while the value of $C_{\max }$ corresponds to $80 \%$ of the maximum contact frequency. As expected, the no strategy (none) and mCT FES values are constant for all app adoption rates. However, detecting a higher percentage of infections (higher IDRs) increases the effectiveness of mCT, resulting in lower FES values for $\mathrm{IDR}_{2}$ and $\mathrm{IDR}_{3}$. At zero app adoption, $\mathrm{CP}$ and CT

(a)

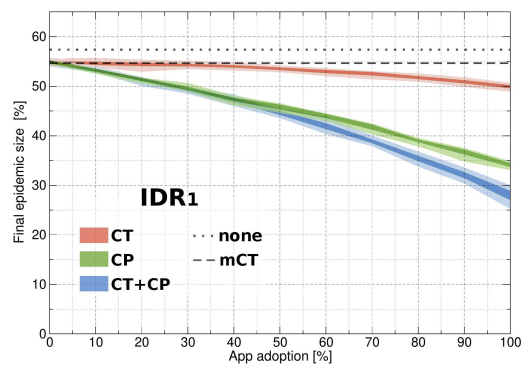

(b)

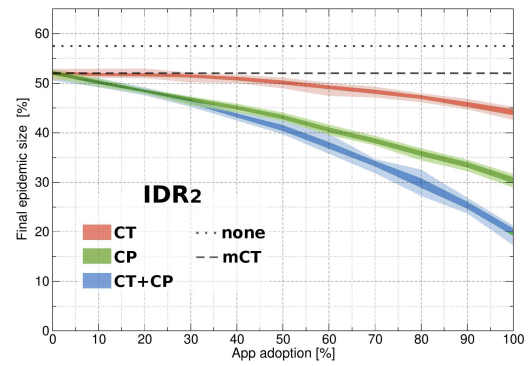

(c)

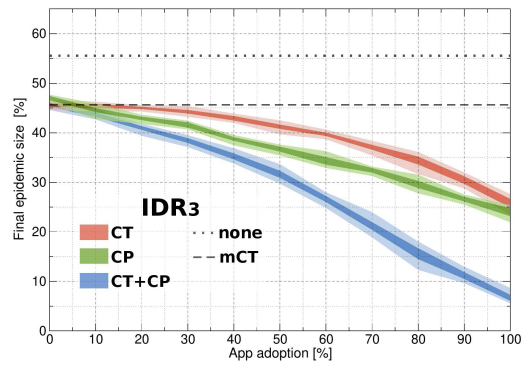

Figure 3. Final epidemic size for CT, CP, and combined CT+CP strategies in three IDR scenarios for varying app adoption percentages. IDR $1, \mathrm{IDR}_{2}$ and $\mathrm{IDR}_{3}$ correspond to $13 \%, 26 \%$ and $37 \%$ overall infections detected. We also plot no strategy (none) or only manual CT (mCT) scenarios as dotted lines for reference. Darker and lighter colored areas show the standard deviation and highest/lowest values obtained from 40 simulations of $1 \times 10^{5}$ agents per point. Results correspond to maximum number of direct contacts $C_{\max }=3.1$ for CP and a delay of $D=3$ days since symptoms onset for CT. 
(a)

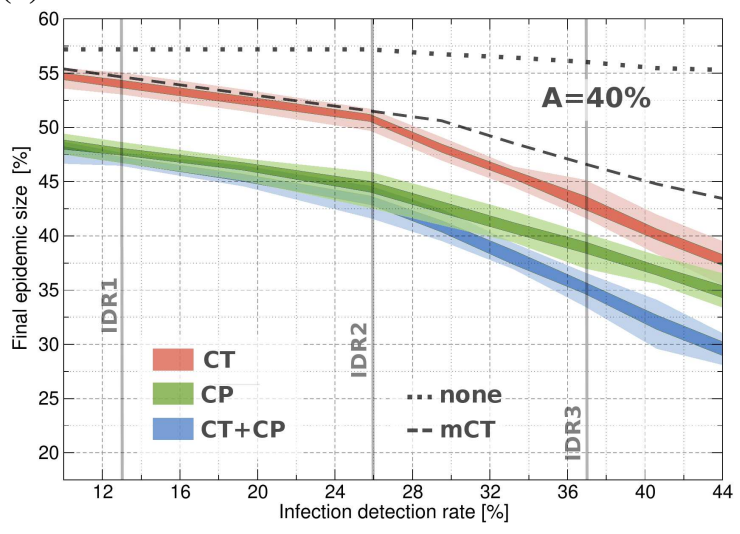

(b)

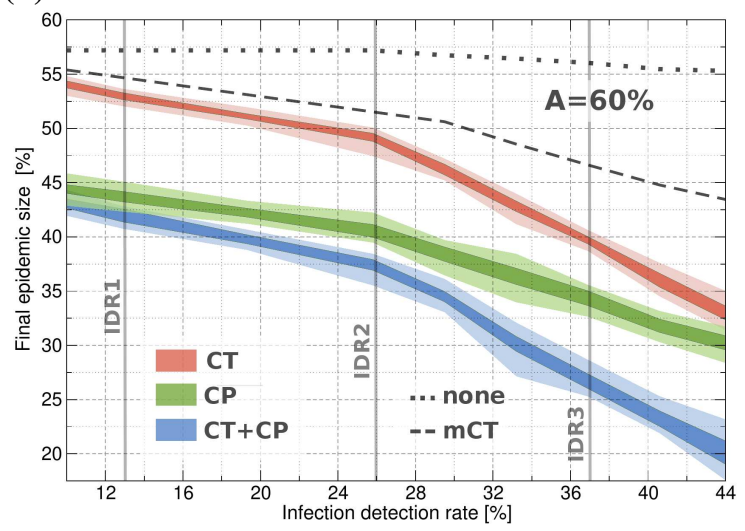

Figure 4. Final epidemic size for $\mathrm{CT}, \mathrm{CP}$, and combined $\mathrm{CT}+\mathrm{CP}$ strategies for varying values of IDR at two fixed app adoption (A) rates. The cases where no strategy (none) or only manual CT (mCT) is applied are plotted as dashed lines for reference (same for both plots). Results correspond to maximum number of direct contacts $C_{\max }=3.1$ for $\mathrm{CP}$ and a delay $D=3$ days since symptoms onset for CT. Darker and lighter coloured areas show the standard deviation and highest/lowest simulation values, respectively.

strategies are as efficient as $\mathrm{mCT}_{\text {. For IDR }}$, CT is almost insensitive to app adoption rates, achieving similar FES values to mCT. However, CP reduces FES by up to $20 \%$ compared with $\mathrm{mCT}$, which suggests that CP is significantly more effective at reducing viral propagation when diagnostic testing is deficient. On the other hand, CT effectiveness increases when detecting all symptomatic agents $\left(\mathrm{IDR}_{2}\right)$ and most notably, when $15 \%$ of asymptomatic agents are also identified (IDR 3$)$. Nevertheless, $\mathrm{CP}$ outperforms $\mathrm{CT}$ in all scenarios, and the combined $\mathrm{CT}+\mathrm{CP}$ strategy proves to be remarkably effective reducing FES from $47-55 \%$ to $26-42 \%$.

To ensure that these results are not restricted to these particular IDR scenarios, we analyze the effectiveness of CT, CP and $\mathrm{CT}+\mathrm{CP}$ under varying values of IDR, as shown in figure 4. Results confirm that low testing efficiency greatly impairs CT, while $\mathrm{CP}$ shows clear improvements with respect to $\mathrm{mCT}$ even at low app adoption rates (40\%). High IDRs allows mCT to have significant effectiveness in reducing viral spread, although it should be noted we are assuming unlimited mCT resources. As a result, $\mathrm{CT}+\mathrm{CP}$ achieves a FES reduction of $23 \%$ with respect to $\mathrm{mCT}$ (for $A=60 \%$ and IDR $=44 \%$ ).

Finally, Table 1 summarizes FES, mortality rate, and $R_{e}$ for the evaluated IDR scenarios. Without applying any mitigation strategy (none), our simulations estimate a FES of $57 \%$ for $\mathrm{IDR}_{1}$ and $\mathrm{IDR}_{2}$ and $56 \%$ for $\mathrm{IDR}_{3}$, suggesting that massive testing has little to no effect without contact tracing. Being insensitive to IDR, CP is capable of reducing $R_{e}$ from 1.52 to 1.36 (for $A=60 \%$ and $\left.\mathrm{IDR}_{1}\right)$. Details shown in figure $\mathrm{S} 11$ reveal that, in contrast with $\mathrm{CT}$, CP lowers $R_{e}$ from the start. Combined $\mathrm{CT}+\mathrm{CP}$ strategies can reduce mortality by $28 \%\left(\mathrm{IDR}_{1}\right), 36 \%\left(\mathrm{IDR}_{2}\right)$ and $56 \%\left(\mathrm{IDR}_{3}\right)$ with respect to $\mathrm{mCT}$.

\section{Discussion}

Although a COVID-19 vaccine is expected to soon be available worldwide as of December 2020 and contagion mitigation will need to persist until herd immunity is achieved, many countries are still seeing increasing amounts of infections. Here we present SERIA, a model which we use to assess CT and CP effectiveness, by contemplating heterogeneous mixing, intricated social interaction patterns and many age-dependant factors such as symptomatic fraction of infections. Our results can offer explanations as to why CT strategies produce conflicting results in different countries, as well as providing compelling evidence that $\mathrm{CP}$ is an appealing complimentary approach to control respiratory viruses such as COVID-19.

Our results confirm that long time delays hinder CT effectiveness ${ }^{5}$, but more importantly, they reveal the strong dependency of CT on infection detection rates (IDR), showing very limited effectiveness in low IDR scenarios. This is specially relevant since more than half of countries are estimated to have IDR values similar or inferior to IDR ${ }_{1}^{11}$ which renders CT almost completely ineffective. Improving IDR implies increasing diagnostic capacity through infrastructure and sufficiently trained personnel, which requires time as well as large economic investments. For underdeveloped and developing countries, this option may not be plausible.

We developed an alternative mitigation strategy, which circumvents these deficiencies, and could be implemented with low economic requirements, which we named contact prevention $(\mathrm{CP})$. Aimed at promoting community self-awareness, self-control and social responsibility, CP leverages digital assets to inform app users regarding their social contact frequency, warning when 


\begin{tabular}{|c|c|c|c|c|c|}
\hline Scenario & $\mathbf{A}$ & Strategy & FES [\%] & $\mathbf{M}[\%]$ & $\mathbf{R}_{e}$ \\
\hline \multirow{8}{*}{$\mathrm{IDR}_{1}$} & \multirow{2}{*}{$0 \%$} & none & 57 & 0.39 & 1.52 \\
\hline & & $\mathrm{mCT}$ & 55 & 0.36 & 1.50 \\
\hline & \multirow{3}{*}{$40 \%$} & $\mathrm{CT}$ & 54 & 0.35 & 1.49 \\
\hline & & $\mathrm{CP}$ & 48 & 0.31 & 1.38 \\
\hline & & $\mathrm{CT}+\mathrm{CP}$ & 47 & 0.31 & 1.38 \\
\hline & \multirow{3}{*}{$60 \%$} & $\mathrm{CT}$ & 53 & 0.34 & 1.46 \\
\hline & & $\mathrm{CP}$ & 44 & 0.29 & 1.36 \\
\hline & & $\mathrm{CT}+\mathrm{CP}$ & 42 & 0.28 & 1.31 \\
\hline \multirow{8}{*}{$\mathrm{IDR}_{2}$} & \multirow{2}{*}{$0 \%$} & none & 57 & 0.39 & 1.52 \\
\hline & & $\mathrm{mCT}$ & 55 & 0.36 & 1.50 \\
\hline & \multirow{3}{*}{$40 \%$} & CT & 51 & 0.33 & 1.44 \\
\hline & & $\mathrm{CP}$ & 45 & 0.29 & 1.36 \\
\hline & & $\mathrm{CT}+\mathrm{CP}$ & 43 & 0.29 & 1.35 \\
\hline & \multirow{3}{*}{$60 \%$} & CT & 49 & 0.32 & 1.42 \\
\hline & & $\mathrm{CP}$ & 40 & 0.27 & 1.32 \\
\hline & & $\mathrm{CT}+\mathrm{CP}$ & 38 & 0.25 & 1.29 \\
\hline \multirow{8}{*}{$\mathrm{IDR}_{3}$} & \multirow{2}{*}{$0 \%$} & none & 56 & 0.37 & 1.51 \\
\hline & & $\mathrm{mCT}$ & 47 & 0.30 & 1.40 \\
\hline & \multirow{3}{*}{$40 \%$} & $\mathrm{CT}$ & 43 & 0.28 & 1.37 \\
\hline & & $\mathrm{CP}$ & 39 & 0.25 & 1.31 \\
\hline & & $\mathrm{CT}+\mathrm{CP}$ & 35 & 0.23 & 1.28 \\
\hline & \multirow{3}{*}{$60 \%$} & CT & 40 & 0.25 & 1.33 \\
\hline & & $\mathrm{CP}$ & 34 & 0.22 & 1.27 \\
\hline & & $\mathrm{CT}+\mathrm{CP}$ & 26 & 0.17 & 1.21 \\
\hline
\end{tabular}

Table 1. Final epidemic size (FES), mortality rate $(M)$ and effective basic reproductive number $\left(\mathrm{R}_{e}\right)$ for three IDR scenarios (average IDR values are given in brackets). Values for different contagion mitigation strategies and different app adoption rates (A) are compared. FES and mortality rates are calculated at the end of each simulation, while $\mathrm{R}_{e}$ values are estimated as averages for days 20 to 25 of each simulation.

social behaviour leads to increased contagion risk.

In contrast to the limitations of $\mathrm{CT}$ analyzed above, $\mathrm{CP}$ proved to remain effective even for low IDRs, which makes it a particularly interesting strategy for countries with limited testing resources. While FES of CT approaches 55\% for low IDRs and $47 \%$ for high IDRs, CP achieves FES in the order of 42-26\% (at 60\% app adoption). Moreover, the combined implementation of CT+CP resulted in $\sim 25 \%$ FES reduction, bringing mortality down by $28 \%-56 \%$ depending on IDR and app adoption rates. CT and $\mathrm{CP}$ techniques proved to be rather orthogonal in their contributions to reduce FES. We explain this by the fact that CT excels at quickly isolating detected symptomatic cases and their close contacts, while CP is able to significantly reduce contagion provoked by asymptomatic and pre-symptomatic carriers. Thus, a joint implementation of $\mathrm{CT}$ and $\mathrm{CP}$ is an appealing approach to mitigate the effects of respiratory virus pandemics.

Moreover, CP presents two further qualitative benefits over CT. One is enhanced privacy of app users, which depending on the $\mathrm{CP}$ app configuration (i.e., identify repeated contacts with the same person in $\mathrm{rCP}$ ) could range from high to full anonymity. The second is long-term game-based habit formation and social conduct modification. The informative notifications from the CP app could provoke profound habit changes that could additionally reduce FES in the long-term, by sustainably making users aware of the risks associated with certain behaviours. Finally, as vaccines are rolled-out, the flexibility of the $C_{\max }$ parameter can be conveniently and controllably increased as the population approaches herd immunity.

A prototype for such a CT+CP application is in development by these authors (and others) in the frame of the ContactAR project.

\section{References}

1. Hale, T., Webster, S., Petherick, A., Phillips, T. \& Kira, B. Oxford covid-19 government response tracker. Blavatnik Sch. Gov. 25 (2020). 
2. Bedford, J. et al. Covid-19: towards controlling of a pandemic. The Lancet 395, 1015-1018 (2020).

3. Braithwaite, I., Callender, T., Bullock, M. \& Aldridge, R. W. Automated and partly automated contact tracing: a systematic review to inform the control of covid-19. The Lancet Digit. Heal. (2020).

4. Hellewell, J. et al. Feasibility of controlling covid-19 outbreaks by isolation of cases and contacts. The Lancet Glob. Heal. (2020).

5. Kretzschmar, M. E. et al. Impact of delays on effectiveness of contact tracing strategies for covid-19: a modelling study. The Lancet Public Heal. 5, e452-e459 (2020).

6. Lee, V. J., Chiew, C. J. \& Khong, W. X. Interrupting transmission of covid-19: lessons from containment efforts in singapore. J. Travel. Medicine 27, taaa039 (2020).

7. Lin, C. et al. Policy decisions and use of information technology to fight coronavirus disease, taiwan. Emerg. infectious diseases 26, 1506 (2020).

8. Garg, S., Bhatnagar, N. \& Gangadharan, N. A case for participatory disease surveillance of the covid-19 pandemic in india. JMIR Public Heal. Surveillance 6, e18795 (2020).

9. Kind, C. Exit through the app store? Patterns 1, 100054 (2020).

10. Klenk, M. \& Duijf, H. Ethics of digital contact tracing and covid-19: who is (not) free to go? Ethics Inf. Technol. DOI: 10.1007/s10676-020-09544-0 (2020).

11. Villalobos, C. Sars-cov-2 infections in the world: An estimation of the infected population and a measure of how higher detection rates save lives. Front. Public Heal. 8, 489, DOI: 10.3389/fpubh.2020.00489 (2020). PMC7545403[pmcid].

12. Yang, W. et al. Estimating the infection-fatality risk of SARS-CoV-2 in new york city during the spring 2020 pandemic wave: a model-based analysis. The Lancet Infect. Dis. DOI: 10.1016/s1473-3099(20)30769-6 (2020).

13. Aleta, A. et al. Modelling the impact of testing, contact tracing and household quarantine on second waves of covid-19. Nat. Hum. Behav. 4, 964-971 (2020).

14. Kaddar, A., Abta, A. \& Alaoui, H. T. A comparison of delayed sir and seir epidemic models. Nonlinear Analysis: Model. Control. 16, 181-190 (2011).

15. Li, R. et al. Substantial undocumented infection facilitates the rapid dissemination of novel coronavirus (sars-cov-2). Science 368, 489-493, DOI: 10.1126/science.abb3221 (2020). https://science.sciencemag.org/content/368/6490/489.full.pdf.

16. Poletti, P. et al. Probability of symptoms and critical disease after sars-cov-2 infection. arXiv preprint arXiv:2006.08471 (2020). 2006.08471.

17. Lauer, S. A. et al. The incubation period of coronavirus disease 2019 (covid-19) from publicly reported confirmed cases: estimation and application. Annals internal medicine 172, 577-582 (2020).

18. Flaxman, S. et al. Report 13: Estimating the number of infections and the impact of non-pharmaceutical interventions on covid-19 in 11 european countries. Imp. Coll. Lond. DOI: https://doi.org/10.25561/77731 (2020).

19. Wölfel, R. et al. Virological assessment of hospitalized patients with covid-2019. Nature 581, 465-469 (2020).

20. Mossong, J. et al. Social contacts and mixing patterns relevant to the spread of infectious diseases. Plos Medicine DOI: https://doi.org/10.1371/journal.pmed.0050074 (2008).

21. He, X. et al. Temporal dynamics in viral shedding and transmissibility of covid-19. Nat. Medicine 26, 672-675, DOI: 10.1038/s41591-020-0869-5 (2020).

22. Montanari, A. Devising and evaluating wearable technology for social dynamics monitoring. Ph.D. thesis, University of Cambridge (2018). DOI: /10.17863/CAM.39687.

23. Danquah, L. O. et al. Use of a mobile application for ebola contact tracing and monitoring in northern sierra leone: a proof-of-concept study. BMC Infect. Dis. 19, 810, DOI: 10.1186/s12879-019-4354-z (2019).

24. Bradshaw, W. J., Alley, E. C., Huggins, J. H., Lloyd, A. L. \& Esvelt, K. M. Bidirectional contact tracing is required for reliable covid-19 control. medRxiv (2020).

25. Kojaku, S., Hébert-Dufresne, L. \& Ahn, Y.-Y. The effectiveness of contact tracing in heterogeneous networks. arXiv preprint arXiv:2005.02362 (2020).

26. Sachdev, D. D. et al. Outcomes of contact tracing in san francisco, california-test and trace during shelter-in-place. JAMA Intern. Medicine (2020). 
27. Kermack, W. O. \& McKendrick, A. G. A contribution to the mathematical theory of epidemics. Proc. royal society london. Ser. A, Containing papers a mathematical physical character 115, 700-721 (1927).

28. Laxminarayan, R. et al. Epidemiology and transmission dynamics of covid-19 in two indian states. Science DOI: 10.1126/science.abd7672 (2020). https://science.sciencemag.org/content/early/2020/09/29/science.abd7672.full.pdf.

\section{Acknowledgments}

The authors acknowledge Universidad Nacional de Córdoba and CONICET, Argentina for funding and support. This research was funded by the "Contact Traceability through the Digital Context of Mobile Devices" (ContactAR) project, awarded by the Agencia Nacional de Promoción de la Investigación, el Desarrollo Tecnológico y la Innovación, under the Ministry of Science, Technology and Productive Innovation of Argentina. We would also like to thank Dr. Rodrigo Castro for careful and critical reading of this manuscript.

\section{Author contributions statement}

All authors concieved the study. GJS designed and programmed the model. JMF concieved the contact prevention strategy. JAF and RQ performed literature research. GJS, JAF and RQ interpreted and analyzed data. All authors contributed to writing the manuscript, and approved the final version for submission. 


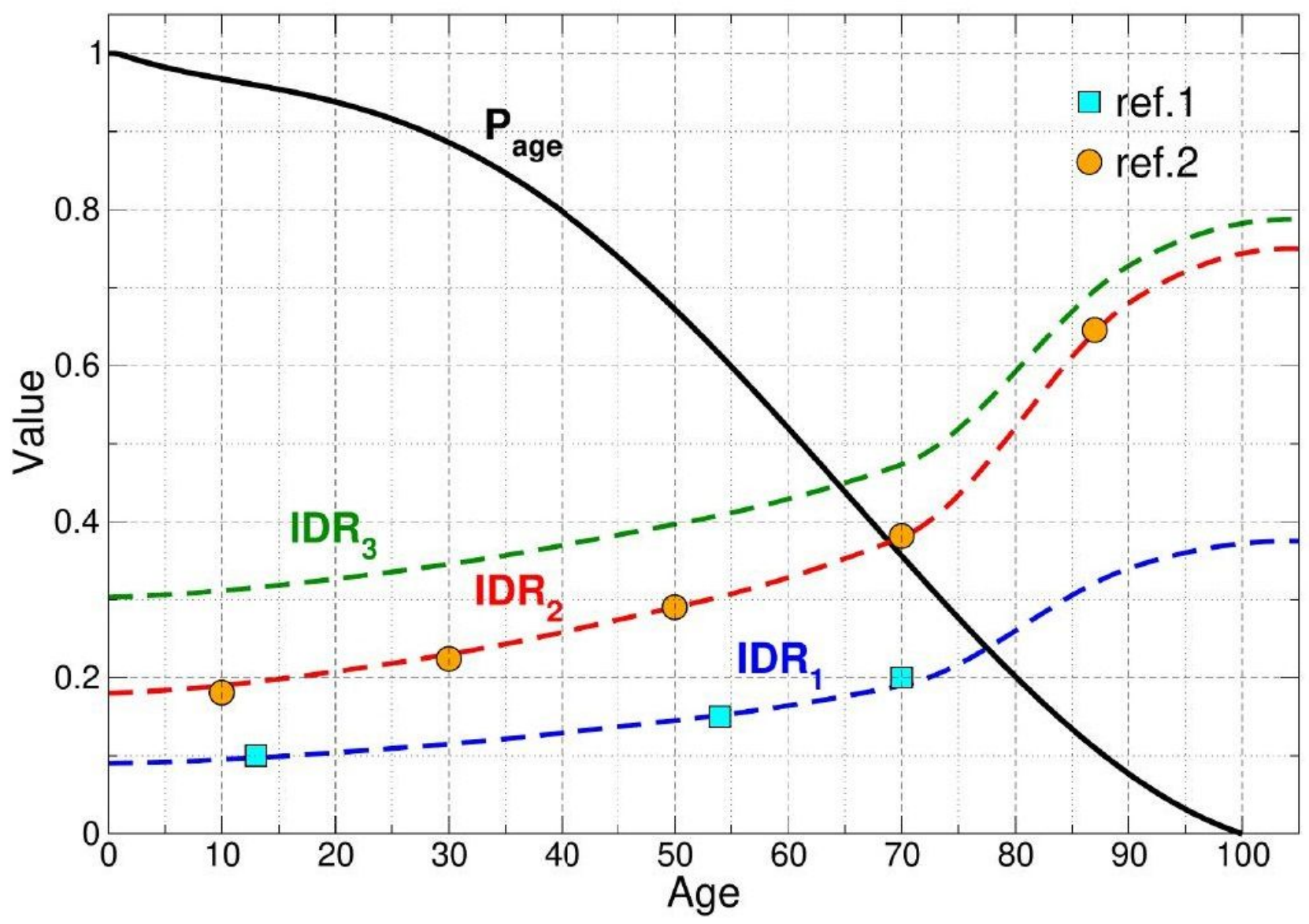

Figure 1

Age distribution function Page and IDR as a function of age for the three scenarios considered in this work: one in which half the symptomatic are detected (IDR1), one in which all of them are (IDR2), and one in which all the symptomatic and $15 \%$ of the asymptomatic (for each age) are detected (IDR3). Ref.1:12 and ref.2:16. 
(a)

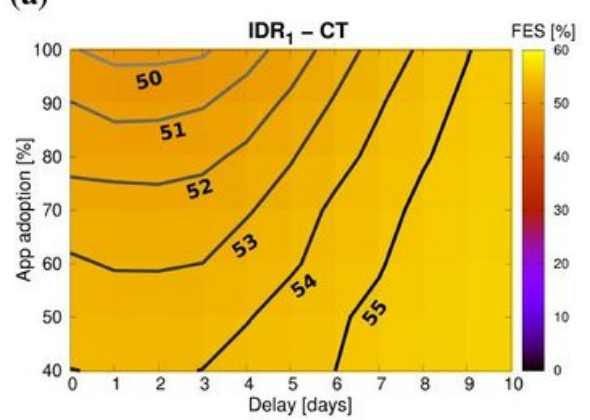

(d)

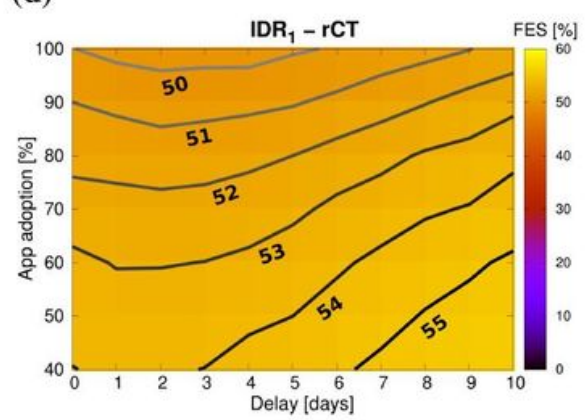

(b)

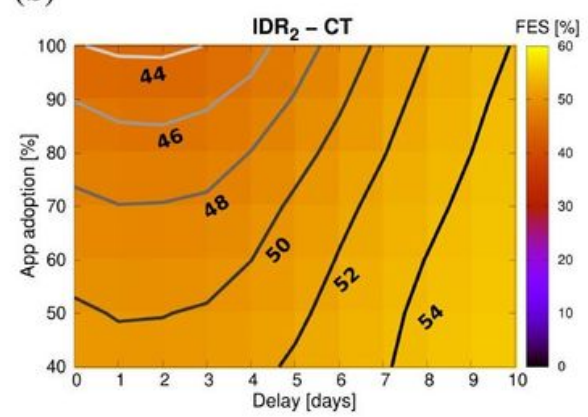

(e)

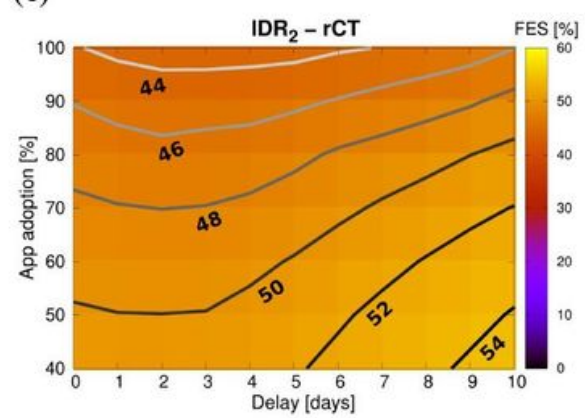

(c)

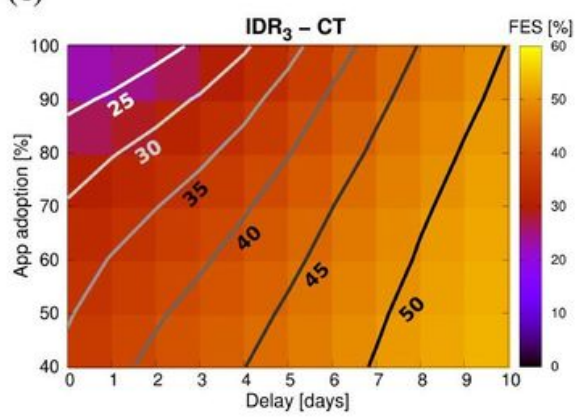

(f)

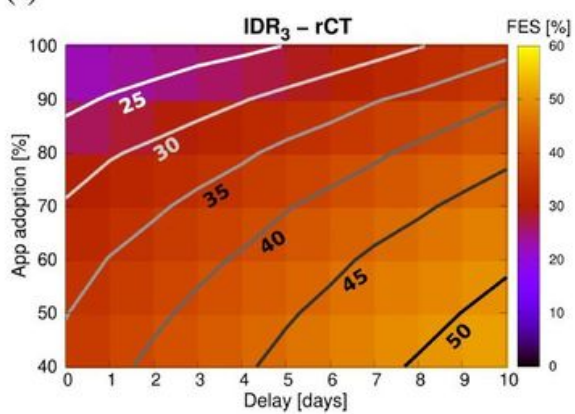

Figure 2

Final epidemic size shown as a heatmap, as a function of CT app adoption and delay (days from symptom onset to contact isolation). The performance of CT (above) and rCT (below) are compared for the three IDR scenarios detailed in figure 1. Contour lines for different FES values are shown. Greater delays, low app adoption, and low IDRs result in high FES values, which indicate low CT effectiveness.

(a)

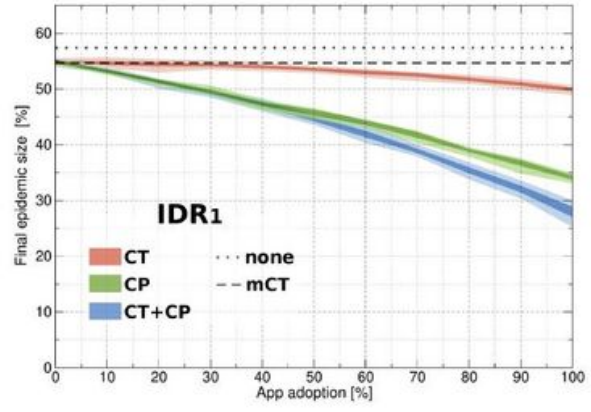

(b)

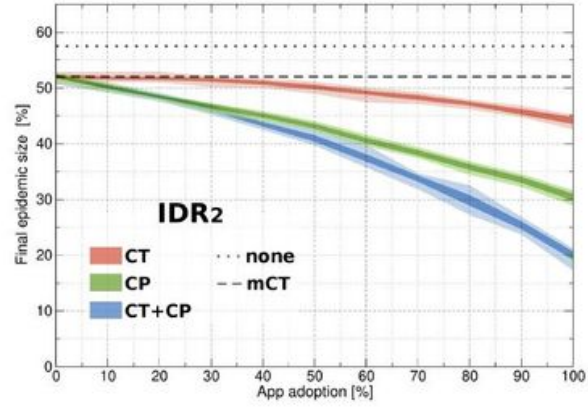

(c)

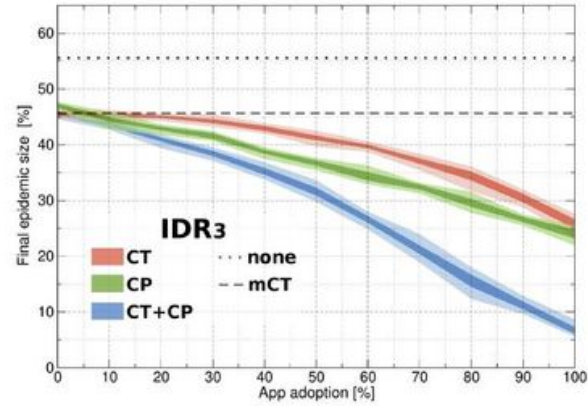

Figure 3

Final epidemic size for CT, CP, and combined CT+CP strategies in three IDR scenarios for varying app adoption percentages. IDR1, IDR2 and IDR3 correspond to $13 \%, 26 \%$ and $37 \%$ overall infections detected. We also plot no strategy (none) or only manual CT (mCT) scenarios as dotted lines for reference. Darker and lighter colored areas show the standard deviation and highest/lowest values obtained from 40 simulations of $1 \times 105$ agents per point. Results correspond to maximum number of direct contactsCmax $=3.1$ for $\mathrm{CP}$ and a delay of $\mathrm{D}=3$ days since symptoms onset for $\mathrm{CT}$. 
(a)

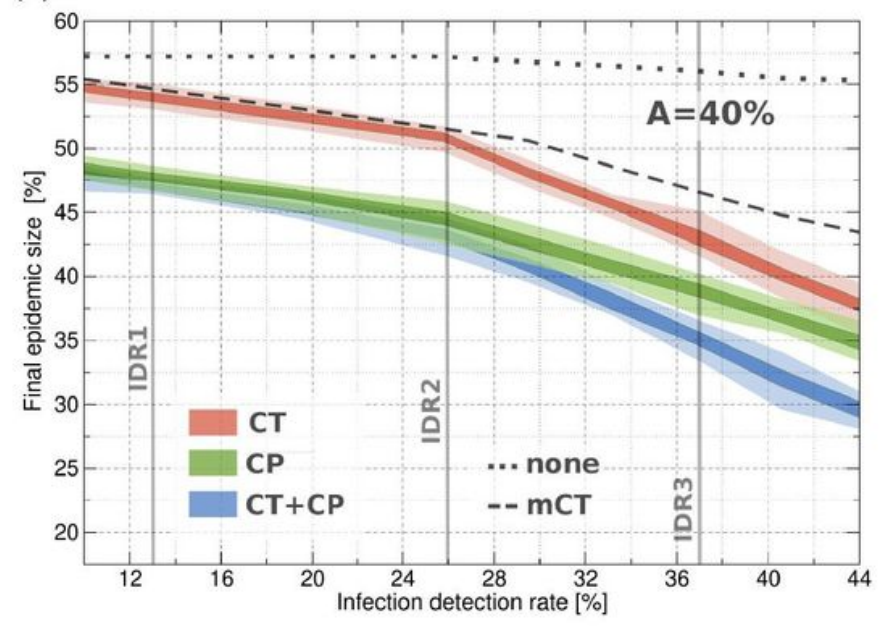

(b)

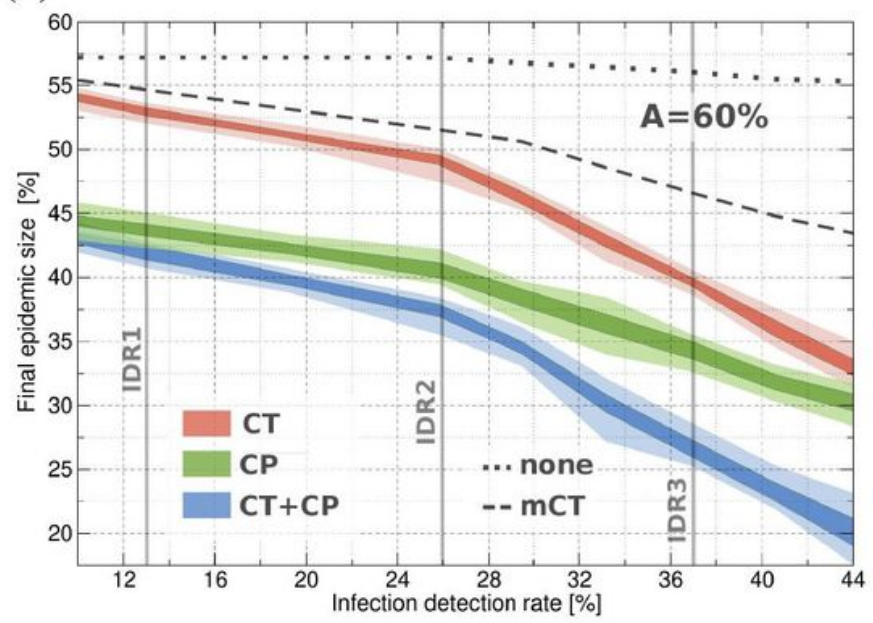

Figure 4

Final epidemic size for $\mathrm{CT}, \mathrm{CP}$, and combined $\mathrm{CT}+\mathrm{CP}$ strategies for varying values of IDR at two fixed app adoption (A) rates. The cases where no strategy (none) or only manual CT ( $\mathrm{MCT}$ ) is applied are plotted as dashed lines for reference (same for both plots). Results correspond to maximum number of direct contactsCmax $=3.1$ for $\mathrm{CP}$ and a delay $\mathrm{D}=3$ days since symptoms onset for $\mathrm{CT}$. Darker and lighter coloured areas show the standard deviation and highest/lowest simulation values, respectively.

\section{Supplementary Files}

This is a list of supplementary files associated with this preprint. Click to download.

- SI.pdf 